\title{
Un sistema, due visioni. Le relazioni monetarie tra Italia e Francia nell'era de Gaulle
}

Un système, deux politiques. Les relations monétaires entre l'Italie et la France pendant l'ère de Gaulle

One System, Two Policies. Monetary Relations between Italy and France during de Gaulle's Era

Daniele Caviglia

\section{OpenEdition}

\section{Journals}

Edizione digitale

URL: http://journals.openedition.org/cei/2951

DOI: 10.4000/cei.2951

ISSN: 2260-779X

\section{Editore}

UGA Éditions/Université Grenoble Alpes

\section{Edizione cartacea}

Data di pubblicazione: 20 avril 2016

Paginazione: 175-188

ISBN: 978-2-84310-323-0

ISSN: 1770-9571 


\title{
UN SISTEMA, DUE VISIONI. LE RELAZIONI MONETARIE TRA ITALIA E FRANCIA NELL'ERA DE GAULLE
}

\author{
Daniele Caviglia \\ Università degli Studi Internazionali di Roma
}

Fino ad oggi le relazioni tra Italia e Francia durante il periodo gollista non sono state oggetto di studi specifici. L'indagine storiografica si è prevalentemente concentrata su singoli aspetti dell'azione internazionale dei due paesi privilegiando soprattutto la prospettiva politico-diplomatica ${ }^{\mathrm{I}}$. In questo saggio ci si propone di offrire un contributo allo studio della diplomazia economico-monetaria, intesa come componente integrante della politica estera. Tale prospettiva consente di notare come anche in questo campo la Francia gollista abbia rappresentato un alleato talvolta problematico per il blocco occidentale impegnato a raccogliere la sfida di Mosca. Le note polemiche di de Gaulle nei riguardi della posizione ricoperta dal dollaro nell'ambito del sistema monetario internazionale ${ }^{2}$

I. Si vedano a tale riguardo i lavori di B. Bagnato, L'Italia e la guerra d'Algeria I954-1962, Soveria Mannelli, Rubbettino, 20I2; Id., La Pira, de Gaulle e il primo colloquio mediterraneo di Firenze, in P. L. Ballini (a cura di), Giorgio La Pira e la Francia. Temi e percorsi di ricerca da Maritain a de Gaulle, Firenze, Giunti, 2005, pp. 99-I34; S. Mourlane, L'Italie et les Italiens sous le regard des diplomates français (1958-1969), "Cahiers de la Méditerranée», n 66, 2003, pp. 279-29I; Id., Le voyage officiel en Italie du général de Gaulle (23-27 juin 1959). De l'usage du rite et du mythe en politique étrangère, "Cahiers de la Méditerranée», $\mathrm{n}^{\circ}$ 77, 2008, pp. 95-IIO; M. Vaïsse, De Gaulle, l'Italie et le projet d'union politique européenne 1958-1963, "Revue d'histoire moderne et contemporaine», $\mathrm{n}^{\circ}$ 42-44, I995, pp. 658-669. Anche il lavoro collettaneo De Gaulle et l'Italie, Roma, École française de Rome, 1997, si concentra in realtà su tematiche specifiche. Di più ampio respiro ma riferito al periodo immediatamente precedente è il volume di I. Russo, Politica estera e 'diplomazia personale'. Fanfani, de Gaulle e le relazioni italo-francesi negli anni Cinquanta, Milano, FrancoAngeli, 2008. Da notare, infine, come negli ultimi anni si sia assistito in Italia a un rinnovato interesse per la figura del Generale, come testimoniano i lavori di R. Brizzi e M. Marchi, Charles de Gaulle, Bologna, Il Mulino, 2008; U. Coldagelli, La Quinta Repubblica. Da de Gaulle a Sarkozy. L'evoluzione di un presidenzialismo extra-costituzionale, Roma, Donzelli, 2009; S. Gentile, Capo carismatico e democrazia: il caso de Gaulle, Milano, FrancoAngeli, I998; G. Quagliariello, De Gaulle, Soveria Mannelli, Rubbettino, 2012.

2. Su questo aspetto cfr. H. Bourguinat, Le général de Gaulle et la réforme du système monétaire international: la contestation manquée de l'hégémonie du dollar, in De Gaulle et son siècle, Parigi, Institut Charles de Gaulle, I992, pp. 90-I03; M. Vaïsse, La grandeur. Politique étrangère du général de Gaulle (1958-I969), Parigi, Fayard, 1998, pp. 396-407. Sull'influenza delle teorie di Jacques Rueff sul presidente francese, C. S. Chivvis, 
finirono infatti per riflettersi non solo sul rapporto con Washington ma anche su quello con i maggiori alleati continentali. In questo contesto l'Italia - che aveva nel frattempo maturato l'adesione ai principi dell'interdipendenza economico-finanziaria, della collaborazione multilaterale e del sostegno al sistema di Bretton Woods - si dovette confrontare con le scelte golliste che mettevano a rischio la coesione transatlantica e la solidarietà intra-europea. Sui temi monetari Roma e Parigi mostrarono in quel periodo di agire sulla base di presupposti difficilmente conciliabili, alimentando in tal modo un clima di tensioni destinate a scaricarsi sulla tenuta complessiva di un sistema che nell'agosto del 1971 sarebbe stato definitivamente soppresso in seguito alla decisione statunitense di sciogliere il dollaro dal vincolo dell'oro.

\section{De Gaulle e la guerra al dollaro}

Nella conferenza stampa del 4 febbraio 1965 de Gaulle apriva un nuovo fronte di contrasto con l'alleato d'oltreoceano ritenuto colpevole di godere di un "privilegio esorbitante» nell'ambito del sistema monetario internazionale. Era l'inizio di un attacco frontale al ruolo del dollaro e agli Stati Uniti, accusati di strumentalizzare le dinamiche monetarie in un quadro di riferimento asimmetrico.

Ce que [les États-Unis] doivent [à l'étranger], ils le lui paient, tout au moins en partie, avec des dollars quil ne tient qu'à eux d'émettre [...]. Cette facilité unilatérale qui est attribuée à l'Amérique contribue à faire s'estomper l'idée que le dollar est un signe impartial et international des échanges, alors quill est un moyen de crédit approprié à un Etat 3 .

La dura presa di posizione mirava da un lato a rimettere in discussione le basi del sistema monetario internazionale in vigore e, dall'altro lato, a ridimensionare il peso di Washington in tale contesto. Di fronte alle incongruenze di un sistema che favoriva la superpotenza americana, de Gaulle respingeva qualsiasi prospettiva di riforma, anche profonda, delle strutture esistenti. Piuttosto, il Generale chiedeva il ritorno al gold standard con l'obiettivo di ripristinare la funzione cardine dell'oro e di ristabilire una simmetria di condizioni per tutti i paesi partecipanti.

Nous tenons donc pour nécessaire que les échanges internationaux sétablissent, comme c'était le cas avant les grands malheurs du monde, sur une base monétaire indiscutable

Charles de Gaulle, Jacques Rueff and French International Monetary Policy under Bretton Woods, "Journal of Contemporary History", vol. 4I, n 4, 2006, pp. 70I-720.

3. Ch. de Gaulle, Discours et messages. Pour l'effort. Août 1962-Décembre 1965, Parigi, Plon, 1970, p. 332.

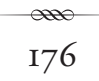


et qui ne porte la marque d'aucun pays en particulier. Quelle base? En vérité, on ne voit pas qu'à cet égard il puisse y avoir de critère, d'étalon, autres que l'or. Eh! oui, l'or, qui ne change pas de nature, qui se met, indifféremment, en barres, en lingots ou en pièces, qui n'a pas de nationalité, qui est tenu, éternellement et universellement, comme la valeur inaltérable et fiduciaire par excellence ${ }^{4}$.

Al di là dell'aspetto propagandistico, de Gaulle era consapevole delle scarsissime possibilità di riattivazione di un sistema aureo ampiamente screditato dall'esperienza negativa del periodo fra le due guerre mondiali. In realtà, l'insistenza sull'urgenza di un superamento degli accordi del I944 era il frutto di una più ampia strategia che aspirava al riequilibrio del rapporto transatlantico. La necessità di ridefinire lo status internazionale del paese traeva origine da una precisa impostazione che individuava negli Stati Uniti, nella Gran Bretagna e nella Francia le uniche nazioni dello schieramento occidentale con responsabilità di carattere 'mondiale' e dotate dell'autonomia derivante dal possesso del nucleare. Muovendo da questo assunto, de Gaulle aveva tentato, subito dopo il ritorno al potere nel 1958, di collocare la Francia su un piede di parità con le nazioni anglosassoni sia con il negoziato diretto che attraverso il rilancio dell'integrazione politica europea. In una prima fase, le iniziative golliste si erano sviluppate all'ombra del memorandum segreto del settembre 1958 con il quale era stata proposta a Washington e a Londra la creazione di un direttorio tripartito che avrebbe dovuto definire le modalità di utilizzazione del nucleare. Si puntava così a superare il monopolio atomico statunitense e a riassorbire il rischio del decouplings in una cornice multilaterale nella quale Parigi avrebbe ottenuto il riconoscimento di un ruolo paritario. Nei mesi successivi la reticenza di Washington e Londra a incamminarsi per un sentiero che minacciava la coesione dell'Alleanza atlantica e impattava sulla special relationship spinse de Gaulle a battere nuove strade. Prese così corpo, favorito dall'acuirsi delle tensioni su Berlino, il proposito gollista di ridare slancio al processo di integrazione europea puntando su una unione politica a guida francese. Pur sfrondato da tutti gli aspetti sovranazionali, il progetto del presidente transalpino rappresentava un tentativo di coagulare il blocco dei Sei in vista di una cooperazione intergovernativa che includeva la politica estera nelle proprie competenze. In tal modo la Francia - che in quel raggruppamento sarebbe stato l'unico paese a possedere armi nucleari — avrebbe di fatto assunto la leadership del continente e, da

4. Ibid.

5. Con questo termine si intende la separazione tra la sicurezza degli Stati Uniti e quella dell'Europa occidentale rispetto alla minaccia di un attacco sovietico. 
questa posizione di forza, avrebbe potuto nuovamente porre in discussione il tema del monopolio statunitense nel quadro atlantico. Progetti europei e riforma dell'Alleanza atlantica si sovrapponevano dunque in una visione incardinata sul recupero del prestigio internazionale della Francia. Di lì a poco, però, i sospetti verso le ambizioni golliste e i timori di ripercussioni negative sulle relazioni con gli anglo-americani indussero i partner europei a frenare le iniziative europeiste di Parigi ${ }^{6}$. Anche il successivo tentativo di ridare slancio ai propri disegni attraverso un rapporto preferenziale con la Repubblica Federale Tedesca ${ }^{7}$ segnò presto il passo, aprendo così la strada alla fase più intransigente della politica gollista prodotta dalla sostanziale indisponibilità di Washington e Londra a rimettere in discussione gli equilibri transatlantici e dalla riluttanza dei partner europei a seguire i disegni europeistici di Parigi.

In questo contesto l'offensiva nei confronti del sistema di Bretton Woods - come pure la decisione del febbraio-marzo 1966 di abbandonare i comandi Nato integrati di Fontainebleau e Rocquencourt - costituì un tentativo di ridimensionare l'egemonia statunitense nell'ambito del blocco occidentale coagulando la crescente insofferenza di molti paesi verso le politiche di bilancio di Washington ${ }^{8}$. Proprio in quegli anni, infatti, l'indisponibilità degli Stati Uniti ad arginare il deflusso di dollari generato dal deficit della bilancia dei pagamenti attraverso politiche fiscali e monetarie di carattere restrittivo aveva cominciato a costituire un nuovo elemento di confronto aspro all'interno del fronte occidentale. Sia nel mondo accademico che in diversi ambienti politici iniziavano a levarsi voci critiche che partivano dall'assunto che spettasse in primo luogo a Washington l'onere di ripristinare l'equilibrio esterno al fine di ridurre gli attacchi speculativi contro il dollaro e quindi i rischi di tenuta dell'intero sistema. Ma mentre negli altri paesi la crescente ostilità nei confronti della politica economica statunitense non si sostanziò mai in atti concreti, Parigi intraprese nel

6. Su queste vicende cfr. D. Caviglia, De Gaulle e il tentativo di spostare l'asse politico europeo: il piano Fouchet, Padova, Cedam, 2000; G.-H. Soutou, Les présidents Charles de Gaulle et Georges Pompidou et les débuts de la coopération politique européenne: du Plan Fouchet au Plan Fouchet light, "Relations internationales", vol. 4, n' 140, 2009, pp. 3-17; Id., Le général de Gaulle, le plan Fouchet et l'Europe, "Commentaire", vol. 4, n ${ }^{\circ}$ 52, 1990, pp. 757-766.

7. Sul tema dei rapporti franco-tedeschi si veda soprattutto G. H. Soutou, L'alliance incertaine. Les rapports politiques et stratégiques franco-allemands, 1954-1996, Parigi, Fayard, 1996.

8. A partire all'incirca dalla metà del decennio il sistema monetario aveva cominciato a evidenziare segnali di squilibrio in coincidenza con l'incremento del fenomeno della speculazione internazionale e dell'aumento costante del deficit della bilancia dei pagamenti americana. In questo quadro, la progressiva liberalizzazione dei movimenti di capitale finì spesso per alimentare tensioni prodotte dalle aspettative degli speculatori sul mercato dei cambi. Le monete giudicate deboli dalle forze del mercato erano così costrette a subire attacchi speculativi che mettevano a rischio un sistema sostanzialmente basato su tassi di cambio fissi. 
corso del ' 65 una vera e propria campagna contro il dollaro cominciando a convertire in oro i dollari detenuti nei forzieri della Banque de France a titolo di riserve. L'iniziativa portò in breve tempo a un mutamento profondo della percentuale delle riserve francesi che alla fine del ' 64 ammontavano al $26,9 \%$ in dollari - pari a I.375 milioni di dollari - e al 73,I\% in oro. Al termine del febbraio ' 66 le riserve in dollari costituivano appena il $\mathrm{I} 2,7 \%$ - pari a 693 milioni di dollari - e quelle in oro erano cresciute fino all' $87,3 \%$. La mossa di Parigi corrispondeva in pratica a una pubblica dichiarazione di sfiducia nei riguardi degli accordi di Bretton Woods che stabilivano la convertibilità delle valute in dollari e l'aggancio di quest'ultimo all'oro, con un'oscillazione massima dell' $1 \%{ }^{\circ}$. La massiccia conversione dei dollari in oro mirava infatti a scardinare uno dei presupposti fondamentali del sistema, dal momento che la condotta della banca centrale francese avrebbe potuto essere seguita da altri istituti di emissione determinando così una pressione insostenibile sulle riserve aurifere della Federal Reserve e costringendo Washington a svalutare di fatto la moneta statunitense ${ }^{\text {Io }}$. Da questo scenario sarebbe emersa, da un lato, l'impraticabilità di un sistema asimmetrico che avrebbe consentito alla Francia di rilanciare la proposta di un ritorno al gold standard e, dall'altro lato, l'inadeguatezza degli Stati Uniti a guidare da una posizione di signoraggio le relazioni monetarie nel mondo occidentale.

Nel complesso si trattava di un atteggiamento caratterizzato da una forte componente contestataria, proprio nel momento in cui il sistema di Bretton Woods si avviava verso una fase di progressivo declino.

\section{L'Italia e la difesa a tutti i costi del sistema di Bretton Woods}

La fase iniziale di adesione al sistema economico e finanziario mondiale post-bellico aveva comportato per l'Italia il superamento di una serie di

9. Una volta stabilita la parità monetaria nessuno l'avrebbe potuta modificare, se non per correggere uno squilibrio fondamentale e dopo aver accolto i suggerimenti del Fondo monetario internazionale. La difesa della parità bilaterale era affidata alle banche centrali che, mediante interventi nel mercato monetario, acquistando o vendendo, avrebbero ricondotto la valuta dentro i parametri. Il meccanismo che ne verrà fuori sarà quello della doppia convertibilità, detto Gold Exchange Standard, in forza del quale la valuta statunitense assumerà il centro del sistema monetario internazionale, diventerà mezzo di pagamento e strumento di riserva.

IO. Qualora gli Stati Uniti avessero accettato di rispettare l'impegno di conversione in oro al prezzo di 35 dollari l'oncia avrebbero depauperato drasticamente le risorse aurifere del paese, peraltro di difficoltosa ricostituzione. Invece in caso di rifiuto il sistema sarebbe automaticamente saltato e il prezzo del dollaro rispetto all'oro sarebbe stato fissato liberamente dal mercato dando luogo ad un aumento che avrebbe a sua volta implicato una rideterminazione di tutti i rapporti di cambio ancorati alla valuta americana. 
ostacoli. La forte impronta statunitense sul nuovo ordine economico internazionale, che sottintendeva un graduale processo d'internazionalizzazione delle economie dei paesi partecipanti, costituiva per la penisola una doppia sfida. Tale impostazione richiedeva infatti non solo lo smantellamento della politica autarchica ma anche la riconversione della mentalità della classe dirigente ${ }^{\text {II }}$ e del ceto imprenditoriale che si erano formati nel Ventennio. L'adesione agli impegni previsti a Bretton Woods, al pari di quella successiva all'Unione europea dei pagamenti ${ }^{{ }^{2}}$, diedero così luogo a un vivace dibattito al punto da apparire «il frutto di una strategia 'giacobina’ portata avanti con determinazione e coerenza da una cerchia ristretta di membri del governo e dall'establishment economico, con in prima fila la Banca d'Italia». Venendo a capo di una serie di resistenze ${ }^{\mathrm{r}}$, la partecipazione dell'Italia ai meccanismi definiti a Bretton Woods si snodò lungo un percorso che negli anni successivi passò attraverso la decisione dell'Oece,

II. A tale proposito appare indicativa la 'rabbia' espressa talvolta da La Malfa "per la sconfortante arretratezza delle culture dei ceti dirigenti, impauriti dai passaggi inevitabili della modernità del paese» (P. Soddu, Ugo La Malfa. Il riformista moderno, Roma, Carocci, 2008, p. 357). Una critica in parte condivisa dallo stesso Andreotti, il quale riconobbe che negli ambienti laici «c'era una sensibilità maggiore, anche perché vi erano esperienze maggiori. Sforza d'altra parte era stato uomo di grandissimo rilievo anche prima del fascismo, poi tutta la sua vita sia negli Stati Uniti che in Paesi europei, Carandini lo stesso per la sua educazione multinazionale; Einaudi; La Malfa, attraverso studi con Mattioli alla Banca Commerciale era molto addentro anche ai problemi della finanza e dell'economia europea. [...] anche Tarchiani era un uomo di vedute larghe che conosceva molto bene anche il mondo anglosassone» (European Oral History, Voices on Europe, INT $579-$ Andreotti Giulio, p. 2).

I2. Sulla Unione europea dei pagamenti, B. Eichengreen, Europe's Post-War Recovery, Cambridge-New York, Cambridge University Press, 1995; Id. (a cura di), Reconstructing Europe's Trade and Payments: The European Payment Union, Manchester, Manchester University Press, 1993; J. J. Kaplan e G. Schleiminger, The European Payments Union: Financial Diplomacy in the 1950s, Oxford, Clarendon Press, 1989.

13. "Una fetta consistente dei partiti che facevano parte della maggioranza e specialmente una larghissima parte del mondo economico erano molto più cauti, se non addirittura contrari a tali scelte e certamente alle prospettive che esse implicavano sul piano dell'effettiva liberalizzazione dell'economia italiana, auspicando tempi e modi più diluiti in vista dell'accettazione definitiva dei principi connessi ad un'economia aperta" (L. Segreto, L'Italia nel sistema economico internazionale: attori e politiche tra anni Cinquanta e Sessanta, in L. Tosi (a cura di), Politica ed economia nelle relazioni internazionali dell'Italia del secondo dopoguerra, Roma, Edizioni Studium, 2002, pp. I82-I83). Perfino un personaggio come Carli aveva in un primo momento espresso qualche perplessità nei confronti di un indirizzo ispirato a un liberismo ortodosso e a un completo abbandono dei controlli sui cambi, mentre Menichella aveva fortemente raccomandato un approccio graduale ai meccanismi multilaterali previsti dagli accordi di Bretton Woods; cfr. F. Petrini, Il liberismo a una dimensione. La Confindustria e l'integrazione europea, 1947-1957, Milano, FrancoAngeli, 2005, pp. 57-58 e p. 77). Anche la maggioranza dell'industria privata nazionale, per quanto cosciente dei vantaggi derivanti da una piena partecipazione alle dinamiche del commercio internazionale, mostrava inquietudine di fronte alla prospettiva di un rapido abbandono di ogni forma di protezione statale; cfr. F. Petrini, Grande mercato, bassi salari: la Confindustria e l'integrazione europea 1947-1964, in P. Craveri e A. Varsori (a cura di), L'Italia nella costruzione europea. Un bilancio storico (1957-2007), Milano, FrancoAngeli, 2009, p. 235. A tale riguardo basti ricordare il dissenso manifestato da alcuni settori della diplomazia e dell'imprenditoria verso il progetto Schuman; cfr. P. L. Ballini e A. Varsori (a cura di), L'Italia e l'Europa (I947-1979), vol. I, Soveria Mannelli, Rubbettino, 2004, pp. 75-76. Per un'analisi del cambiamento dell'atteggiamento degli industriali italiani nei confronti del processo d'integrazione europea, vedi R. Ranieri, L'integrazione europea e gli ambienti economici italiani, in R. H. Rainero (a cura di), Storia dell'integrazione europea, vol. I, Roma, Marzorati, I997, pp. 285-329. 
nel novembre 1949, di liberalizzare almeno il 50\% delle voci d'importazione dei paesi membri e la creazione, l'anno successivo, dell'Unione europea dei pagamenti. Contrariamente ai timori di molti le due iniziative - alle quali si aggiunse lo stimolo commerciale della guerra di Corea e l'applicazione delle proposte di Ugo La Malfa in favore della riduzione unilaterale dei dazi del I0\% e della liberalizzazione di circa il 90\% delle merci importate dall'area Oece - favorirono le esportazioni italiane verso le destinazioni europee e anche le importazioni dalle aree del dollaro e della sterlina. In questa cornice l'Italia era risultata «tra i paesi che maggiormente avevano tratto giovamento dal sistema di Bretton Woods e soprattutto dalla liberalizzazione economica e commerciale» ${ }^{\mathrm{T}}$, al punto che nelle considerazioni finali relative al 1959 il neo governatore della Banca d'Italia, Guido Carli, poteva affermare che «il progresso compiuto dall'Italia era risultato maggiore di quello dei paesi industrializzati dell'Europa occidentale e dell'America» ${ }^{15}$.

I lusinghieri risultati conseguiti nel corso degli anni del 'miracolo economico' ${ }^{16}$ contribuirono a integrare il sistema monetario internazionale nell'approccio complessivo del paese verso le dinamiche della guerra fredda. Laddove il Patto atlantico garantiva ai paesi europei la sicurezza e li esimeva da investimenti in campo militare troppo onerosi e potenzialmente inflazionisti, gli accordi di Bretton Woods assicuravano quella cornice entro la quale si era articolata la ripresa post-bellica. Muovendo da tali presupposti non sorprende pertanto che la diplomazia italiana avesse ben presto compendiato le esigenze politiche di coesione del blocco occidentale e le opportunità di risanamento economico in una linea di condotta di convinto sostegno al sistema in vigore e alla valuta americana che ne costituiva l'architrave.

I4. L. Segreto, L'Italia nel sistema economico internazionale, cit., p. I94.

I5. Settori strategici come quelli della chimica e della fabbricazione dei mezzi di trasporto avevano conosciuto aumenti della produzione nell'ordine del 50\%, giustificando in tal modo «l'apprezzamento che dei progressi [dell'Italia] si fa[ceva] in ambienti stranieri» (Considerazioni finali del Governatore della Banca d'Italia [CF], I960, pp. 323-324).

16. Per un'analisi del 'miracolo economico' che attribuisce il ruolo trainante ai consumi interni, più che alle esportazioni, si veda R. Petri, Storia economica d'Italia. Dalla Grande guerra al miracolo economico (I9I8-1963), Bologna, Il Mulino, 2002, pp. I88-217. Sullo stesso tema ma con un occhio all'influenza dell'adozione della tecnologia americana nel processo di produzione italiano, vedi V. Zamagni, Un'analisi critica del 'miracolo economico italiano': nuovi mercati e tecnologia americana, in E. Di Nolfo, R. H. Rainero e B. Vigezzi (a cura di), L'Italia e la politica di potenza in Europa (1950-1960), Milano, Marzorati, 1992, pp. 393-422. Sulle ricadute socio-culturali della crescita economica, vedi S. Lanaro, Storia dell'Italia repubblicana. Dalla fine della guerra agli anni Novanta, Venezia, Marsilio, 1992, pp. 223-306. Sul governo dell'economia nel periodo del boom, vedi P. Craveri, La Repubblica dal I958 al I992, Milano, TEA, I996, pp. 85-92. Una particolare attenzione al ruolo della Banca d'Italia si trova in F. Cotula e J. C. Martinez Oliva, Stabilità e sviluppo dalla liberazione al 'miracolo economico' italiano, in F. Cotula, M. De Cecco e G. Tonioni (a cura di), La Banca d'Italia. Sintesi della ricerca storica I893-I960, Laterza, Bari, 2003, pp. 415-493. 
Dopo le iniziali perplessità, l'Italia aveva presto adottato una impostazione che prevedeva «ogni sforzo per collaborare ad un [...] migliore funzionamento e, in prospettiva, ad un [...] ampliamento» ${ }^{17}$ degli accordi di Bretton Woods. In tal senso la distanza dall'approccio maturato dalla Francia non poteva essere più evidente se si pensa che, a poco più di due mesi dalla conferenza stampa di de Gaulle, la Farnesina tornava a confermare che «la posizione italiana di solidarietà nei confronti del dollaro si [era] manifestata innanzi tutto sul piano politico, dato il ruolo [...] riconosci[uto] al dollaro nell'assetto mondiale», ma non aveva mancato di concretizzarsi «anche sul piano più strettamente monetario». Mentre quindi la Francia intraprendeva una battaglia per minare la centralità della valuta americana, l'Italia ribadiva il suo impegno in difesa del sistema in vigore che implicava, «direttamente o indirettamente», l'adozione di misure volte «a rafforzare la posizione del dollaro» ${ }^{18}$. Per le autorità italiane questo atteggiamento derivava dalla percezione che il nuovo sistema monetario rappresentava uno dei cardini delle nuove relazioni transatlantiche imperniate sui principi dell'interdipendenza e della cooperazione internazionale. Quando quindi all'inizio degli anni 1960 iniziò a farsi strada il fenomeno della speculazione internazionale, agevolato dalle «dimensioni straordinarie dei movimenti di fondi a breve termine», risultò quasi naturale che il governatore della Banca d'Italia si trovasse in prima fila nel caldeggiare una cooperazione tra banche centrali in grado «di reagire con prontezza anche ad attacchi di notevole portata». I suggerimenti di Carli in favore di una collaborazione "più stretta e più variamente articolata che in passato ${ }^{19}$ erano infatti in linea con l'elaborazione di un orientamento incline alla stabilizzazione del quadro economico-finanziario. Nell'insieme quello delle autorità italiane era un approccio fortemente antirevisionista nella misura in cui escludeva «innovazioni radicali» ${ }^{20}$ e puntava invece al rafforzamento delle strutture esistenti. Per l'Italia la coincidenza tra l'efficiente funzionamento degli accordi di Bretton Woods e la tutela delle esigenze nazionali non poteva essere più completa tanto che il governatore, in un appunto trasmesso al ministro del Tesoro Roberto Tremelloni, sottolineava che «con il contribuire alla difesa di un valido e per ora insostituibile

17. L. Segreto, L'Italia nel sistema economico internazionale, cit., p. 194.

18. Appunto del Mae, Il problema dell'oro e del dollaro, Roma, s. d., Archivio centrale dello Stato (Acs), Carte Moro (cm), busta 35, sotto-fascicolo 2, Visita dell'On. Presidente del Consiglio e dell'On. Min. degli Esteri a Washington (20-2I aprile 1965) e sfasc. 20, Il problema dell'oro e del dollaro.

I9. CF, I96I, pp. 299-300.

20. Ivi, p. 299. 
sistema monetario internazionale, non si compi[va] opera di generosità e altruismo, ma si segu[iva] una politica di illuminato autointeresse» ${ }^{21}$.

\section{I diritti speciali di prelievo: due visioni a confronto}

All'inizio degli anni 1960 il deterioramento del quadro monetario internazionale accelerò il confronto all'interno del blocco occidentale tra $\mathrm{i}$ sostenitori dello status quo e i fautori del cambiamento. In particolare, l'affacciarsi di consistenti ondate speculative sui principali mercati internazionali iniziò a stimolare l'attenzione sulla sostenibilità del sistema monetario internazionale, soprattutto in ambito accademico ${ }^{22}$. In questa situazione, la decisione dell'estate del ' 63 dei ministri delle Finanze e dei governatori delle banche centrali del Gruppo dei Dieci ${ }^{23}$ di avviare una serie di consultazioni sui problemi riguardanti la liquidità internazionale determinò un deciso cambio di passo. L'iniziativa indicava l'emergere di una volontà comune dei paesi più industrializzati di prendere in considerazione l'ipotesi di un progetto di riforma dei meccanismi di creazione delle riserve mondiali aprendo così una fase di riflessione sull'efficienza complessiva del sistema monetario internazionale. Ben presto però lo studio in comune si tramutò in un duro confronto nel cui ambito finirono per emergere le differenti impostazioni che caratterizzavano Roma e Parigi.

Da una parte gli Stati Uniti, appoggiati dal Canada e dalla Gran Bretagna, desideravano una riforma che contemplasse la creazione di riserve addizionali o di credito internazionale allo scopo di «agevolare il processo

2I. Appunto di Carli per Tremelloni, Roma, 27 settembre 1962, Archivio storico della Banca d'Italia (Asbi), Direttorio Carli (Dc), cart. 62, fasc. I, sfasc. Io. Al fine di consolidare il sistema, l'Italia era pronta a fare la sua parte. Come spiegò il governatore ai colleghi del Fmi durante la riunione annuale del i960, l'Italia era un paese che, potendo vantare dei "progressi in direzione della soluzione di alcuni problemi strutturali», era in grado di agire "con piena coscienza dei doveri verso la comunità internazionale derivanti dalla sua nuova posizione» (Discorso di Carli alla Assemblea annuale del Fmi, Washington, 28 settembre 1960, in G. Carli, Scritti e Conferenze (I959-I963), vol. I, Roma, Banca d'Italia, I969, p. 42). Per una ricostruzione più dettagliata della politica monetaria internazionale dell'Italia in questo periodo, vedi D. Caviglia, La diplomazia della lira. L'Italia e la crisi del sistema di Bretton Woods (1958-1973), Milano, FrancoAngeli, 2013.

22. Basti a tale riguardo richiamare le note riflessioni di Robert Triffin anticipate in due articoli apparsi nel 1959: The Return to Convertibility: 1926-I93I and 1958-? or, Convertibility and the Morning After, "Quarterly Review», marzo 1959, pp. 3-57, e Tomorrow's Convertibility: Aims and Means of International Monetary Policy, "Quarterly Review», giugno 1959, pp. I3I-200. Le tesi furono poi ulteriormente sviluppate nel volume Gold and the Dollar Crisis: The Future of Convertibility, New Haven (Conn.), Yale University Press, 1960. Sulla figura e sull'opera di Triffin si veda fra gli altri: M. Dumoulin, C. Ferrant, O. Lefebvre e J. Sloover (a cura di), Robert Triffin, conseiller des princes. Souvenirs et documents, Bruxelles, Peter Lang, 2010.

23. Il Gruppo dei Dieci non era altro che l'emanazione diretta del General Agreement to Borrow al quale partecipavano Belgio, Francia, Italia, Repubblica Federale Tedesca, Olanda, Svezia, Canada, Giappone, Gran Bretagna e Stati Uniti. 
di aggiustamento dei futuri squilibri, di non alterare il finanziamento dei deficit passati, di aggiungere riserve allo stock mondiale, di non rimpiazzare i dollari al momento in uso ${ }^{24}$. In quest'ottica la posizione della Francia, volta a scalzare il ruolo dominante del dollaro e a promuovere una disciplina simmetrica sul deficit, divenne il contraltare su cui si snodò l'intero negoziato sui diritti speciali di prelievo. Dal canto suo l'Italia dovette contemperare la solidarietà intracomunitaria con la preservazione dei legami con gli Stati Uniti, trovando la soluzione al difficile dilemma nell'esercizio di una costante opera di mediazione.

Già nel corso della riunione di Parigi del Gruppo dei Dieci (I5-16 giugno 1964) si erano manifestate le prime avvisaglie di uno scontro che avrebbe presto costretto gli altri paesi europei a un difficile equilibrismo. In quella occasione era stata la richiesta del rappresentante britannico Reginald Maudling di chiarire i termini del concetto di «sorveglianza multilaterale», scaturito dall'intensificazione della cooperazione monetaria internazionale, a provocare un primo accenno di confronto. Venendo incontro ai timori del ministro inglese, il sottosegretario statunitense al Tesoro, Robert Roosa, spiegò che i «delegati non avevano ritenuto possibile o saggio arrivare a delle formulazioni precise» dal momento che tutti ritenevano che fosse «meglio far evolvere il processo nel tempo». Dato che lo scopo principale della collaborazione consisteva nel rapido ed efficace intervento diretto a fronteggiare una situazione di emergenza, secondo Roosa non esisteva alcun obbligo di consultazione preventiva per l'uso delle facilitazioni in vigore ma solo l'impegno a comunicare agli altri paesi il volume totale dell'operazione. Mentre Witteveen per l'Olanda e Colombo per l'Italia «avevano sostenuto» l'impostazione statunitense, Giscard d'Estaing si era soffermato sul fatto che la formula adottata sulla sorveglianza multilaterale era «molto meno strutturata e molto meno concreta di quanto la Francia aveva originariamente proposto e desiderato». Per il ministro delle Finanze transalpino il processo avrebbe dovuto operare «sulle stesse linee della formulazione della politica dei redditi sul piano nazionale: guardare alle conseguenze delle azioni e delle decisioni individuali sull'insieme e cercare di raggiungere un consenso sulle linee generali da seguire» ${ }^{25}$. Nelle osservazioni di Giscard si palesava la preoccupazione del governo francese di evitare che ai crediti forniti ai paesi in deficit attraverso i vari accordi

24. Letter from the Chairman of the Task Force on Foreign Economic Policy (Kaysen) to President Johnson, Washington, 25 novembre 1964, Foreign Relations of the United States (FRUS), 1964-I968, vol. VIII, International Monetary and Trade Policy, doc. I8.

25. Telegram from the Embassy in France to the Department of State, Parigi, I6 giugno 1964, FRUS, 1964I968, vol. VIII, International Monetary and Trade Policy, doc. 9. 
di natura bilaterale e multilaterale non corrispondesse alcun tipo di controllo da parte dei prestatori, con conseguenze negative sia sulla disciplina budgetaria dei riceventi che sulle varie economie nazionali. Era questa la logica di fondo che aveva animato la controproposta francese, nota come Collective o Composite riserve unit (Cru), avanzata senza successo proprio in quel periodo. Preso atto dell'impossibilità di ottenere il consenso degli altri paesi su un progetto che mirava a creare un nuovo mezzo di riserva al di fuori del Fondo monetario internazionale e a ridurre il ruolo del dollaro, de Gaulle invitò il governo a «evitare di impegnarsi in discussioni relative alla definizione di nuovi sistemi di credito visto che si tratt[ava] di un esercizio teorico senza seria possibilità di riuscita ${ }^{26}$. Per il Generale, infatti, qualsiasi ipotesi di riforma del sistema monetario internazionale restava subordinata al miglioramento del deficit statunitense, tanto più che i progetti in discussione fra i Dieci apparivano «lontani» dalla concezione francese in favore del ritorno all'oro e rischiavano, «per la combinazione dell'uso del dollaro e delle nuove unità di riserva, di condurre a una pericolosa inflazione mondiale» ${ }^{27}$.

Solo il rischio di un isolamento in ambito comunitario e di un concomitante peggioramento dei rapporti con gli Stati Uniti indussero la Francia a non abbandonare le trattative. Su queste basi, in occasione dell'incontro del 25 e 26 gennaio 1967 tra i rappresentanti del Gruppo dei Dieci e i direttori esecutivi del $\mathrm{Fmi}$, il governo transalpino apparve più disposto a prendere in considerazione l'ipotesi dei diritti speciali di prelievo ${ }^{28}$. D'altra parte, nel successivo vertice dei ministri delle Finanze dei Sei il governo francese ebbe modo di constatare ancora una volta l'isolamento crescente che posizioni sostenute da Parigi producevano in ambito comunitario. L'Italia, in particolare, si era opposta alle proposte avanzate dal ministro dell'Economia e delle Finanze, Michel Debré, «non ritene[ndo] soddisfacente un sistema che fac[esse] prevalente affidamento sull'afflusso di

26. Nota di J. Chabrun e J. Dromer per de Gaulle, Parigi, 3I gennaio 1966, Archives de la Présidence de la République - Charles de Gaulle (Apr-Cdg), Conseils restreints des Affaires économiques, 5 AG I/28, d. "Conseil restreint du 25 février 1966. Problèmes monétaires internationaux», sd. «État des négociations».

27. Consiglio ristretto, Apr-Cdg, Conseils restreints des Affaires économiques, 5 AG I/28, d. «Conseil restreint du 25 février 1966. Problèmes monétaires internationaux», Relevé de décisions.

28. La riunione, tenutasi all'Aja (30 marzo-I aprile 1967) registrò un certo consenso intorno alla formula dei diritti speciali di prelievo, ai quali vennero attribuite una serie di caratteristiche proprie delle unità di riserva (apertura di un conto separato e quindi di un finanziamento con tecniche che li avrebbero distinti dalle risorse generali del Fmi, trasferimento diretto secondo regole specifiche piuttosto che attraverso uno scambio di valute per mezzo del Fondo). Da notare che il ministro degli Esteri, Maurice Couve de Murville, aveva definito i diritti speciali di prelievo «intellettualmente seducenti» ma anche "poco realisti» (M. Couve de Murville, Une politique étrangère 1958-1969, Parigi, Plon, 1971, p. 150). 
oro alle riserve monetarie perché ciò [avrebbe] presuppo[sto] un aumento del prezzo dell'oro» ${ }^{29}$. Secondo quanto sostenuto dal ministro del Tesoro, Emilio Colombo, per produrre effetti rilevanti il rialzo del prezzo dell'oro avrebbe dovuto essere "di dimensioni notevoli», con il rischio di generare «un aumento esagerato del volume delle liquidità incondizionate in rapporto ai bisogni attuali»; oppure, l'operazione avrebbe dovuto essere ripetuta a intervalli ravvicinati nel tempo, correndo in questo caso il pericolo «di scuotere la fiducia dei detentori di mezzi di riserva diversi dall'oro e quindi di incoraggiare la conversione di questi mezzi in oro con il risultato ultimo di diminuire anziché aumentare il livello delle liquidità internazionali». Di fronte a questa chiusura, condivisa dagli altri paesi Cee, era emersa «la possibilità della modifica dell'atteggiamento francese» alla quale era seguito l'esame da parte dei Sei di un aumento delle quote di partecipazione dei paesi comunitari al Fmi «per accrescere la presenza europea in quella istituzione» ${ }^{30}$. Una volta svanita ogni realistica prospettiva di imporre la propria linea e di fronte al pericolo di vedere crescere l'isolamento rispetto ai partner comunitari, la Francia si era quindi accontentata di assecondare l'accoglimento di tutti quei suggerimenti che miravano al riconoscimento del potenziale economico dei Sei originato dai successi della ricostruzione postbellica e dai progressi del mercato comunitario. L'ammorbidimento della linea gollista — favorito dall'impegno dell'Italia e della Repubblica Federale Tedesca ${ }^{31}$ — permise di riassorbire il dissenso di Parigi e di cementare il fronte dei Sei in una prospettiva di confronto negoziale con gli anglo-americani. Su queste basi, i ministri delle Finanze poterono procedere all'approvazione del rapporto del Comitato monetario della Cee in cui era prevista la creazione di diritti di prelievo automatici, separati da quelli già esistenti nel Fmi, utilizzabili

29. La bocciatura delle proposte francesi era motivata principalmente dall'iniqua ripartizione dei profitti implicita nel recupero del vecchio sistema aureo: «Sul piano concettuale, un ritorno puro e semplice al Gold Standard sarebbe non soltanto anacronistico, ma anche dannoso per vari motivi. In primo luogo, come l'esperienza insegna, tale sistema è del tutto rigido e le sue conseguenze sono risentite soprattutto nei paesi meno sviluppati e debitori. In secondo luogo, la produzione mondiale dell'oro su cui dovrebbe essere esclusivamente basato un sistema di Gold Standard non sarebbe sufficiente per tenere il passo con l'espansione e le esigenze del commercio internazionale. In terzo luogo, un aumento del prezzo dell'oro arreca profitti differenziati secondo il capriccio delle riserve di tale metallo disponibili nei vari paesi: tra i favoriti vi sarebbe l'URSS, non certo l'Italia" (Appunti, "Il problema dell'oro e del dollaro", Acs, cm, b. 35, fasc. 20, sfasc. 2 "Visita dell'On. Presidente del Consiglio e dell'On. Ministro degli Affari esteri a Washington (20-2I aprile 1965))».

30. Colloquio Moro-Humphrey, Palazzo Chigi, 3I marzo 1967, Acs, cm, b. 44, fasc. 238 «Colloqui 1967".

31. L'importanza della mediazione italiana era stata riconosciuta anche dal direttore generale del Fmi, PierrePaul Schweitzer, il quale aveva affermato che «esiste[va] la possibilità di trascinare i francesi dietro pressione dei tedeschi e degli italiani. I francesi resisteranno e sarà come trascinare un mulo, ma de Gaulle sarà aperto a una limitata pressione» (Memorandum of Conversation, Washington, 26 maggio 1967, Frus, 1964-1968, vol. VIII, International Monetary and Trade Policy, doc. I25). 
secondo regole prestabilite e direttamente trasferibili tra le autorità monetarie dei paesi partecipanti. In aggiunta, i Sei si accordarono affinché gli emendamenti relativi ai diritti di prelievo procedessero parallelamente a una più profonda revisione delle regole del Fondo che includesse la restrizione delle condizioni di accesso ai diritti di prelievo nelle credit tranches e la semplificazione della definizione delle parità e delle unità di conto del Fmi. Soprattutto, i paesi comunitari ufficializzarono la presa di posizione in favore di una maggioranza qualificata - pari all' $85 \%$ dei voti rafforzata dalla partecipazione di almeno la metà dei maggiori paesi creditori — $\mathrm{da}$ applicare alle decisioni riguardanti la creazione di riserve addizionali e i mutamenti nelle quote del Fmi detenute dai singoli membri. La consapevolezza che tali rivendicazioni «non erano tanto [il frutto] dell'intransigenza francese quanto del desiderio della Comunità europea nell'insieme di avere una voce più forte» e «di ottenere un ruolo più dominante»" ${ }^{2}$ nell'ambito delle operazioni ordinarie di un Fmi riformato, indussero Washington a lasciare cadere le ultime resistenze anche sugli aspetti non ancora pienamente chiariti dei meccanismi decisionali e della revisione dello statuto del Fondo.

Il Gruppo dei Dieci, riunitosi a Londra alla fine di agosto, poteva così concordare uno schema, denominato "Outline of a Contingency Plan", che, dopo essere stato trasmesso e fatto proprio dal Comitato dei governatori del Fmi, veniva allegato alla risoluzione approvata al vertice annuale di Rio de Janeiro del 29 settembre 1967. Dopo più di tre anni di tortuosi negoziati il progetto, qualificato come "Outline of a Facility Based on Special Drawing Rights in the Fund», definì finalmente i contorni dei nuovi mezzi di riserva chiamati a tappare le falle del sistema di Bretton Woods. Il ricompattamento del fronte occidentale attorno ad uno schema condiviso non poteva però cancellare l'impressione che le differenti impostazioni con cui la Francia e i suoi principali alleati guardavano al futuro delle relazioni monetarie internazionali avrebbero finito per minare la solidità del sistema.

32. Current Economic Developments, Washington, 2 agosto 1967 , cit. In effetti, se i rappresentanti francesi non mancavano occasione per manifestare la "grande importanza» che per loro rivestiva il parallelismo tra approvazione dei Dsp e riforma dello statuto del Fmi (cfr. Verbale della $2 \mathrm{O}^{\mathrm{a}}$ seduta del Comitato dei Governatori delle Banche Centrali degli Stati membri della Comunità Economica Europea, Basilea, II settembre 1967, <www. ecb.europa.eu/ecb/history/archive/agendas/html/index.it.html >) anche gli altri cinque paesi della Comunità finirono per convergere su questa posizione. 


\section{Conclusioni}

A partire dalla metà degli anni 1960 i temi monetari avevano rappresentato un fattore di contrasto tra Parigi e Roma, anche se nel periodo a ridosso della crisi finale del sistema si registrò un certo riavvicinamento. Lungi però dall'essere il frutto di una visione comune, il cambiamento apparve piuttosto derivare da una serie di considerazioni negative. La sensazione di un progressivo allontanamento fra le due sponde dell'Atlantico ${ }^{33}$, la maturazione di una posizione italiana più critica nei confronti del deficit statunitense ${ }^{34}$, la comune diffidenza verso il Piano Werner che avviava la strutturazione di una identità monetaria europea ${ }^{35}$ e la delusione per la scarsa solidarietà tra i partner comunitari, costituirono elementi in gran parte condivisi dai due paesi in un momento di difficile transizione verso la ridefinizione di un nuovo sistema monetario internazionale. Anche l'evoluzione del quadro interno dei due paesi concorse a diminuire le distanze. Da una parte, la crisi del franco sul finire del '68 e l'uscita di scena di de Gaulle ridussero fortemente la carica polemica dell'impostazione di Parigi; dall'altra parte, la crescente instabilità politica e sociale trasformarono l'Italia in un attore di secondo piano preoccupato di restare agganciato al treno dell'Europa e non più in grado di proporre soluzioni efficaci ai problemi delle relazioni monetarie internazionali. Erano dunque più le dinamiche interne e la crescita dell'insofferenza nei riguardi di Washington e dell'Unione economica e monetaria a riavvicinare Roma e Parigi piuttosto che la condivisione dei principi di una riforma dell'ordine monetario internazionale che appariva sempre più lontana all'orizzonte in un panorama segnato dall'impronta dell'unilateralismo.

33. Si vedano a tale riguardo le Considerazioni finali svolte da Carli nel I969; cfr. CF, I969, p. 344.

34. Per una valutazione critica della politica statunitense di quel periodo si veda il seguente giudizio di Gavin: «Fra il 1958 e il 197I, il principale obiettivo della politica economica estera degli Stati Uniti fu quello di trovare una strada per controllare il deficit della bilancia dei pagamenti americana e frenare la perdita di oro dal Tesoro statunitense. Molte delle politiche che furono messe in atto o prese in considerazione [...] erano in conflitto con gli scopi più ampi della politica estera, della strategia e della politica economica nazionale degli Usa. Questa contrapposizione generava enormi tensioni sia all'interno del governo americano che con gli alleati occidentali» (F. J. Gavin, Gold, Dollars \& Power. The Politics of International Monetary Relations, 1958-1971, Chapel Hill e Londra, The University of North Carolina Press, 2004, p. 7).

35. Sulle perplessità della Banca d'Italia nei riguardi del piano Werner si vedano A. Verde, Carli e l'economia internazionale dei primi anni Settanta, «Nuova Storia Contemporanea», a. IX, n 2, marzo-aprile 2005, pp. 7I-72; L. Segreto, L'Italie et le plan Werner, in Comité pour l'histoire économique et financière de la France (a cura di), Le rôle des ministères des Finances et de l'Économie dans la construction européenne (1957-1978), t. I e II, Paris, Ministère des Finances, 2002, pp. 27-51. 